Copyright $($ IFAC Large Scale Systems: Theory and Applications,

\title{
OVERLAPPING GUARANTEED COST CONTROL FOR UNCERTAIN DISCRETE-TIME SYSTEMS
}

\author{
Lubomír Bakule *, José Rodellar ${ }^{* *}$ and Josep M. Rossell *** \\ * Institute of Information Theory and Automation, Academy of Sciences \\ of the Czech Republic, 18208 Prague 8, Czech Republic \\ (bakule@utia.cas.cz). \\ ** Department of Applied Mathematics III, Universitat Politècnica de \\ Catalunya (UPC), Campus Nord, C-2, 08034-Barcelona, Spain \\ (jose.rodellar@upc.es). \\ *** Department of Applied Mathematics III, Universitat Politècnica de \\ Catalunva (UPC), 08240-Manresa, Spain \\ (josep.maria.rossell@upc.es)
}

\begin{abstract}
Robust overlapping guaranteed cost control problem is presented for a class of state-delayed discrete-time systems. Unknown arbitrarily time-varying uncertainties with given known bounds are considered. Conditions preserving closed-loop systems expansioncontraction relations including the equality of bounds of costs are proved. A linear matrix inequality (LMI) delay independent approach is used for control design in the expanded space. The results are specialized on the overlapping decentralized control design. A numerical illustrative example is supplied. Copyright $@ 2004$ IFAC
\end{abstract}

Keywords: Large-scale systems, discrete-time systems, time-delay, decentralized control, overlapping.

\section{INTRODUCTION}

The disjoint decomposition of the global system is not reasonable if the resulting subsystems are strongly connected. This is particularly true if the subsystems share some common parts. The overlapping subsystems may be in fact weakly connected although disjoint subsystems are not. A systematic way of overlapping decompositions starts with the expansion of the original system. To carry out the expansioncontraction process correctly, certain conditions must be satisfied to ensure that solutions of the original system are included in solutions of the expanded system. A general mathematical framework for this process

\footnotetext{
1 This work was supported in part by the Czech Ministry of Education. Youth and Sport (MSMT) under Grant IP04LA209, by the Academy of Sciences of the Czech Republic (AVCR) under Grant A2075304, and by the Committee for Science and Technology (ClCYT) of Spain under Grant DPI2002-04018-C02-01
}

has been called the Inclusion Principle (Ikeda and Šiljak 1980), (Ikeda et al. 1981), (Ikeda et al. 1984), (Šiljak 1991). The Inclusion Principle has been applied to different classes of systems and problems as illustrated for instance in (Bakule et al. 2000a), (Bakule et al. 2000b), (Bakule et al. 2002b), (Šiljak and Stipanović 2000).

The paper deals with the expansion-contraction relations for a class of uncertain state-delayed discretetime systems with quadratic performance index. The concept of quadratic guaranteed cost controller is introduced for instance in (Yu 1997), (Xie and Soh 1993), (Guan et al. 1999), (Jiang et al. 2000). The delay independent control design procedure is included. It is based on the LMI approach by (Yu et al. 2000) and it is used as a control design tool. The main results concern the conditions on the expansion-contraction relations between closed-loop systems including the requirement on the equality of bounds on costs. The 
results are given in the form of conditions on the complementary matrices. The specialization of these results on the decentralized overlapping control design is given including an illustrative example.

To the authors knowledge, the Inclusion Principle has not been extended up to now using the concept of guaranteed cost control for the considered class of uncertain state-delayed problems.

\section{PROBLEM FORMULATION}

\subsection{The Inclusion Principle}

Consider an uncertain discrete-time system with state delay, denoted by $\mathbf{S}$, and described by the state equation:

$$
\begin{gathered}
x(k+1)=\left(A+\Delta_{A}\right) x(k)+\left(A_{d}+\Delta_{A_{d}}\right) x(k-d) \\
+\left(B+\Delta_{B}\right) u(k), \\
x(k)=\dot{\phi}(k),-d \leqslant k \leqslant 0,
\end{gathered}
$$

where $d$ is a positive integer denoting the time delay and $\phi(k)$ is an initial value at $k$. The cost function associated with this system has the form

$$
J(x, u)=\sum_{k=0}^{\infty} x^{T}(k) Q^{*} x(k)+u^{T}(k) R^{*} u(k) .
$$

Similarly, consider a system $\tilde{\mathbf{S}}$ in the form

$$
\begin{gathered}
\tilde{x}(k+1)=\left(\tilde{A}+\Delta_{\tilde{A}}\right) \tilde{x}(k)+\left(\tilde{A}_{d}+\Delta_{\tilde{A}_{d}}\right) \tilde{x}(k-d) \\
+\left(\tilde{B}+\Delta_{\tilde{B}}\right) u(k), \\
\tilde{x}(k)=\tilde{\phi}(k),-d \leqslant k \leqslant 0 .
\end{gathered}
$$

The cost function associated with this system is

$$
\tilde{J}(\tilde{x}, \tilde{u})=\sum_{k=0}^{\infty} \tilde{x}^{T}(k) \tilde{Q}^{*} \tilde{x}(k)+u^{T}(k) \tilde{R}^{*} u(k) .
$$

The vectors $x(k) \in \mathbb{R}^{\mathrm{n}}, u(k) \in \mathbb{R}^{\mathrm{m}}$, and $\tilde{x}(k) \in \mathbb{R}^{\mathrm{n}}$ are the states and the inputs of $\mathbf{S}$ and $\tilde{\mathbf{S}}$ at the time instant $k$, respectively. Suppose $n \leqslant n$. The matrices $A, B, A_{d}, Q^{*}$, $R^{*}$ and $\tilde{A}, \tilde{B}, \tilde{A}_{d}, \tilde{Q}^{*}, \tilde{R}^{*}$ are constant with appropriate dimensions. $\Delta_{A}, \Delta_{B}, \Delta_{A_{d}}, \Delta_{\tilde{A}}, \Delta_{\bar{B}}$ and $\Delta_{\tilde{A}_{d}}$ are realvalued matrices of uncertain parameters. Uncertainties are assumed to be norm-bounded uncertainties as follows:

$$
\begin{aligned}
& \left(\begin{array}{lll}
\Delta_{A} & \Delta_{B} & \Delta_{A_{d}}
\end{array}\right)=D \Delta\left(\begin{array}{lll}
E_{1} & E_{2} & E_{d}
\end{array}\right), \\
& \left(\begin{array}{llll}
\Delta_{\tilde{A}} & \Delta_{\tilde{B}} & \Delta_{\tilde{A}_{d}}
\end{array}\right)=\tilde{D} \tilde{\Delta}\left(\begin{array}{lll}
\tilde{E}_{1} & \tilde{E}_{2} & \tilde{E}_{d}
\end{array}\right) .
\end{aligned}
$$

$D, E_{1}, E_{2}, E_{d}, \tilde{D}, \tilde{E}_{1}, \tilde{E}_{2}, \tilde{E}_{d}$ are known constant matrices and $\Delta, \tilde{\Delta}$ are unknown arbitrarily timevarying matrices satisfying $\Delta^{T} \Delta \leqslant I, \tilde{\Delta}^{T} \tilde{\Delta} \leqslant I$. Denote $x(k)=x(k ; \phi(k), u(k))$ and $\tilde{x}(k)=\tilde{x}(k ; \tilde{\phi}(k), u(k))$ the formal solutions of (1) and (3) for given inputs $u(k)$ (Bakule et al. 2002a).

Suppose the standard relations between the states given within the Inclusion Principle. It means the systems $\mathbf{S}$ and $\tilde{\mathbf{S}}$ given in (1) and (3) are related by the following linear transformations

$$
\tilde{x}(k)=V x(k), \quad x(k)=U \tilde{x}(k),
$$

where $V$ and $U$ are constant full-rank matrices of appropriate dimensions.

Definition 1. A system $\tilde{\mathbf{S}}$ includes the system $\mathbf{S}$, denoted by $\tilde{\mathbf{S}} \supset \mathbf{S}$, if there exists a pair of constant matrices $(U, V)$ such that $U V=I_{n}$ and for any initial state $\phi(k)$ and any fixed input $u(k)$ of $\mathbf{S}$, $x(k ; \phi(k), u(k))=U \tilde{x}(k ; V \phi(k), u(k))$ for all $k$.

Moreover, impose an additional assumption requiring the equality of the cost functions (2) and (4) corresponding to the systems $\mathbf{S}$ and $\tilde{\mathbf{S}}$. It means $J(x, u)=\tilde{J}(V x, u)$.

Definition 2. A control law $u(k)=\tilde{K} \tilde{x}(k)$ for $\tilde{\mathbf{S}}$ is contractible to $u(k)=K x(k)$ for $S$ if the choice $\tilde{\phi}(k)=V \phi(k)$ implies $K x(k ; \phi(k), u(k))=\tilde{K} \tilde{x}(k ; V \phi(k), u(k))$ for all $k$ any initial state $\phi(k)$ and any fixed input $u(k)$ of $\mathbf{S}$.

Suppose given a pair of matrices $(U, V)$. Then, the matrices $\tilde{A}, \Delta_{\tilde{A}}, \tilde{A}_{d}, \Delta_{\tilde{A}}, \tilde{B}, \Delta_{\tilde{B}}, \tilde{Q}^{*}$ and $\tilde{R}^{*}$ can be described as

$$
\begin{aligned}
\tilde{A} & =V A U+M, & \Delta_{\tilde{A}} & =V \Delta_{A} U, \\
\tilde{A}_{d} & =V A_{d} U+M_{d}, & \Delta_{\tilde{A}_{d}} & =V \Delta_{A_{d}} U, \\
\tilde{B} & =V B+N, & \Delta_{\tilde{B}} & =V \Delta_{B}, \\
\tilde{Q}^{*} & =U^{T} Q^{*} U+M_{Q^{*}}, & \tilde{R}^{*} & =R^{*}+N_{R^{*}},
\end{aligned}
$$

where $M, M_{d}, N, M_{Q^{*}}$ and $N_{R^{*}}$ are so called complementary matrices. The complementary matrices play a fundamental role in the context of the Inclusion Principle, because they allow to construct different expanded systems with desirable properties (Bakule et al. 2000a), (Bakule et al. 2000b), (Bakule et al. 2001).

Definition 1 can be rewritten in terms of complementary matrices in an equivalent form which can be deduced by extending known results (Bakule et al. 2000a). The following proposition presents two important cases of sufficient conditions for $\tilde{\mathbf{S}} \supset \mathbf{S}$ using the complementary matrices.

Proposition 3. Consider the systems $\mathbf{S}$ and $\tilde{\mathbf{S}}$ as given in (1) and (3), respectively. A system $\tilde{\mathbf{S}} \supset \mathbf{S}$ if

$$
\begin{aligned}
& \text { a) } \quad M V=0, \quad M_{d} V=0, \quad N=0 \quad \text { or } \\
& \text { b) } U M=0, \quad U M_{d}=0, \quad U N=0 \text {. }
\end{aligned}
$$

Remark. If $M_{d}=0$ in (8), then $a$ ) and b) correspond to particular cases within the Inclusion Principle called restrictions and aggregations, respectively, (Ikeda et al. 1981), (Ikeda and Siljak 1986), (Šiljak 1991).

\subsection{Guaranteed Cost Control}

To simplify, consider only the system (1), (2). We deal with the control design problem of a state memoryless feedback control law such that the corresponding closed-loop system is quadratically stable and guarantees an upper bound of a quadratic cost function. 
Definition 4. Suppose the system (1) with the associated cost function (2). A state feedback control law $u(k)=K x(k)$ is said to be a quadratic guaranteed cost control law if the resulting closed-loop system

$$
\begin{aligned}
\mathbf{S}_{\mathrm{C}}: x(k+1) & =\left[A+B K+\Delta_{A}+\Delta_{B} K\right] x(k) \\
& +\left(A_{d}+\Delta_{A_{d}}\right) x(k-d)
\end{aligned}
$$

is quadratically stable and the cost function (2) of the the closed-loop system satisfies

$$
J=\sum_{k=0}^{\infty} x^{T}(k)\left(Q^{*}+K^{T} R^{*} K\right) x(k) \leqslant J_{0}
$$

for all admissible uncertainties (5) and any delay. $J_{0}$ is some given constant.

Now, introduce an another definition which is equivalent to Definition 4, but it is better suitable for the control design using the LMI approach presented in the subsection 2.3 .

Definition 5. A control law $u(k)=K x(k)$ is said to be a quadratic guaranteed cost control with an associated cost matrix $P>0$ for the system (1), (5) and the cost function (2) if there exists a semipositive-definite matrix $W$ such that

$$
\left(\begin{array}{ll}
H_{1} & H_{2} \\
H_{2}^{T} & H_{3}
\end{array}\right)<0
$$

where

$$
\begin{gathered}
H_{1}=\left[A+B K+\Delta_{A}+\Delta_{B} K\right]^{T} P\left[A+B K+\Delta_{A}+\Delta_{B} K\right] \\
-P+W+Q^{*}+K^{T} R^{*} K, \\
H_{2}=\left[A_{d}+\Delta_{A_{d}}\right]^{T} P\left[A+B K+\Delta_{A}+\Delta_{B} K\right] \\
H_{3}=\left[A_{d}+\Delta_{A_{d}}\right]^{T} P\left[A_{d}+\Delta_{A_{d}}\right]-W .
\end{gathered}
$$

\subsection{LMI Approach}

There are available different approaches to compute quadratic guaranteed cost control laws such as the Riccati equations approach or the LMI approach (Guan et al. 1999), (Jiang et al. 2000), (Xie and Soh 1993), (Yu 1997). A delay independent linear matrix inequality approach is selected to design a linear state memoryless feedback controller guaranteeing that the system is quadratically stable with a desired upper bound on the quadratic cost function. The following proposition gives sufficient conditions to get a guaranteed cost control law (Yu et al. 2000). To simplify, the result is presented only for the system (1), (2), but it evidently holds also for the expanded system.

Proposition 6. Suppose the system (1) with its corresponding cost function (2). If there exist symmetric positive definite matrices $X, T$ a matrix $Y$ and a constant $\varepsilon>0$ such that the following linear matrix inequality

$$
\left(\begin{array}{cccccc}
-X+\varepsilon D D^{T} & A X+B Y & A_{d} X & 0 & 0 & 0 \\
X A^{T}+Y^{T} B^{T} & -X+T & 0 & X E_{1}^{T}+Y^{T} E_{2}^{T} & X & Y^{T} \\
X A_{d}^{T} & 0 & -T & X E_{d}^{T} & 0 & 0 \\
0 & E_{1} X+E_{2} Y & E_{d} X & -\varepsilon I & 0 & 0 \\
0 & X & 0 & 0 & -\left(Q^{*}\right)^{-1} & 0 \\
0 & Y & 0 & 0 & 0 & -\left(R^{*}\right)^{-1}
\end{array}\right)<0
$$

holds, then $u(k)=K x(k)=Y X^{-1} x(k)$ is a guaranteed cost controller of system (1) and the corresponding closed-loop value of the cost function satisfies $J \leqslant J_{0}$, where

$$
J_{0}=x^{T}(0) X^{-1} x(0)+\sum_{k=-d}^{-1} \phi^{T}(k) X^{-1} T X^{-1} \phi(k) .
$$

Remark. The relation $X=P^{-1}, T=P^{-1} W P^{-1}$ holds. It relates the matrices $X, T$ in Proposition 6 and $P, W$ in Definition 5.

\subsection{The Problem}

Suppose given a state-delayed discrete-time uncertain system by $\mathbf{S}(1),(5)$ with an associated cost function $J$ by (2). Consider an expanded system $\tilde{\mathbf{S}}$ by (3), (5) with an associated cost function $J$ by (4). Suppose that $\tilde{\mathbf{S}} \supset \mathbf{S}$ holds by Definition 1. The specific goals are as follows:

- Derive conditions under which the relation $\tilde{\mathbf{S}}_{\mathbf{C}} \supset \mathbf{S}_{\mathrm{C}}$ holds.

- Derive conditions under which the relations $\tilde{\mathbf{S}}_{\mathbf{C}} \supset \mathbf{S}_{\mathrm{C}}$ and $J=\tilde{J}$ hold simultaneously. Use the concept of quadratic guaranteed cost control.

- Specialize the global system results into decentralized control design setting.

- Supply these results with a numerical example.

Derive all the above results in terms of complementary matrices. Use the delay independent LMI approach to compute the required gain matrices.

\section{MAIN CONTRIBUTION}

\subsection{Controllers and Closed-Loop Systems}

The Inclusion Principle has be applied for the analysis and control design of different classes of dynamic systems following various objectives. The control design is usually performed for a larger expanded system $\tilde{\mathrm{S}}$ so that this step is followed by the consequent contraction and implementation of the control law into the smaller system $\mathbf{S}$. This approach is effective mainly when considering decentralized controller design.

Definition 2 presents the conditions under which a control law designed in the expanded system $\tilde{\mathbf{S}}$ can be contracted and implemented into the initial system 
S. However, these requirements do not guarantee that the closed-loop system $\tilde{\mathbf{S}}_{\mathrm{C}}$ includes the closed-loop system $\mathbf{S}_{\mathbf{C}}$ in the sense of the Inclusion Principle, i.e. $\tilde{\mathbf{S}}_{\mathrm{C}} \supset \mathbf{S}_{\mathbf{c}}$. Therefore, the conditions guaranteeing this requirement must be given. They are presented using the complementary matrices by the following proposition.

Proposition 7. Consider the systems (1) and (3) such that $\tilde{\mathbf{S}} \supset \mathbf{S}$. Suppose that $u(k)=\tilde{K} \tilde{x}(k)$ is a contractible control law designed for $\tilde{\mathbf{S}}$. If $M V=0, N=0$ and $M_{d} V=\mathbf{0}$, then $\tilde{\mathrm{S}}_{\mathrm{C}} \supset \mathrm{S}_{\mathrm{C}}$.

Proof. Suppose $\tilde{\mathbf{S}} \supset \mathbf{S}$ and consider $u(k)=\tilde{K} \tilde{x}(k)$ a contractible control law designed in $\tilde{\mathbf{S}}$. The corresponding state matrix of the uncertain closed-loop expanded system $\tilde{\mathbf{S}}_{\mathrm{C}}$ is given by

$$
\begin{aligned}
\tilde{x}(k+1) & =\left[\tilde{A}+\Delta_{\tilde{A}}+\left(\tilde{B}+\Delta_{\tilde{B}}\right) \tilde{K}\right] \tilde{x}(k)+\left(\tilde{A}_{d}+\Delta_{\tilde{A}_{d}}\right) \tilde{x}(k-d) \\
& =\tilde{A}_{c} \tilde{x}(k)+\left(\tilde{A}_{d}+\Delta_{\tilde{A}_{d}}\right) \tilde{x}(k-d) .
\end{aligned}
$$

Similar expression can be obtained for the closedloop system $\mathbf{S}_{\mathrm{C}}$. Consider the relation between the state matrices of the closed-loop systems $\tilde{\mathbf{S}}_{\mathrm{C}}$ and $\mathbf{S}_{\mathrm{C}}$. The relation $\tilde{A}_{C}=V A_{C} U+M_{C}$ implies $M_{C}=M+N \tilde{K}+$ $V B \tilde{K}-V B \tilde{K} V U+V \Delta_{B} \tilde{K}(I-V U)$, where $M_{C}$ is a complementary matrix to be determined. Since $\tilde{\mathbf{S}}_{\mathrm{C}} \supset \mathbf{S}_{\mathrm{C}}$ is desired, the conditions $U M_{C}^{i} V=0, i=1,2, \cdots, \tilde{n}$, must be satisfied. Imposing these requirements and using equations (7), we can prove that $M V=0, N=0$ is a sufficient condition so that the relation $U M_{C}^{i} V=0$ holds for all $i=1,2, \cdots, \tilde{n}$. On the other hand, if $M_{d}$ in (7) verifies $M_{d} V=0$, then the general conditions of the Inclusion Principle $U M_{d l}^{i} V=0$ holds for all $i=1,2, \cdots, \tilde{n}$. Finally, by Definition 2 the contracted gain matrix $K$ is given by $K=\tilde{K} V$.

Remark. By using the conditions $M V=0, N=0$ and $M_{d} V=0$, any control law $u(k)$ designed in $\tilde{\mathbf{S}}$ is always contractible to S (Ikeda et al. 1981), (Ikeda and Šiljak 1986), (Šiljak 1991).

\subsection{Cost Functions}

Consider the systems (1), (3) with associated cost functions (2), (4), respectively. Now, impose the assumption on the equality of the cost functions $J$ and $\tilde{J}$. A sufficient condition for this requirement presents the following proposition.

Proposition 8. Consider the systems (1) and (3) such that $\tilde{\mathbf{S}} \supset \mathbf{S}$. Suppose that $u(k)=\tilde{K} \tilde{x}(k)$ is a contractible control law designed in $\tilde{\mathbf{S}}$. If $V^{T} M_{Q^{*}} V=0$ and $N_{R^{*}}=0$, then $J=\tilde{J}$.

Proof. Consider the cost functions $J$ and $\tilde{J}$ given in (2) and (4), respectively. Consider $u(k)=\tilde{K} V x(k)$ a contracted control law. Substitute $\tilde{Q}^{*}=U^{T} Q^{*} U+$ $M_{Q^{*}}, \tilde{R}^{*}=R^{*}+N_{R^{*}}$ in (4) and compare the obtained cost function with the cost function given in (2). It it straightforward to prove that $V^{T} M_{Q^{*}} V=0, N_{R^{*}}=0$ is a sufficient condition so that $J=\tilde{J}$.

Theorem 9. Consider the systems (1) and (3) with their corresponding cost functions (2) and (4), respectively. Suppose that $M V=0, N=0, M_{d} V=0, V^{T} M_{Q} V=0$ and $N_{R^{*}}=0$ hold. Then, $u(k)=\tilde{K} \tilde{x}(k)$ is a quadratic guaranteed cost controller with an associated cost matrix $\tilde{P}>0$ for the system $\tilde{\mathbf{S}}$ if and only if $u(k)=K x(k)=\tilde{K} V x(k)$ is the contracted quadratic guaranteed cost controller with an associated cost matrix $P>0$ for $\mathbf{S}$ and $J_{0}=\tilde{J}_{0}$. The matrices $P$ and $W$ in (12), if they exist, are related by $P=V^{T} \tilde{P} V, W=V^{T} \tilde{W} V$.

Proof. Consider $u(k)=\tilde{K} \tilde{x}(k)$ a contractible control law for the expanded system $\tilde{\mathbf{S}}$. By Definition 5, suppose there exists a semipositive-definite matrix $\tilde{W}$ such that

$$
\left(\begin{array}{ll}
\tilde{H}_{1} & \tilde{H}_{2} \\
\tilde{H}_{2}^{T} & \tilde{H}_{3}
\end{array}\right)<0
$$

where

$$
\begin{gathered}
\tilde{H}_{1}=\left[\tilde{A}+\tilde{B} \tilde{K}+\Delta_{\tilde{A}}+\Delta_{\tilde{B}} \tilde{K}\right]^{T} \tilde{P}\left[\tilde{A}+\tilde{B} \tilde{K}+\Delta_{\tilde{A}}+\Delta_{\tilde{B}} \tilde{K}\right] \\
-\tilde{P}+\tilde{W}+\tilde{Q}^{*}+\tilde{K}^{T} \tilde{R}^{*} \tilde{K}, \\
\tilde{H}_{2}=\left[\tilde{A}_{d}+\Delta_{\tilde{A}_{d}}\right]^{T} \tilde{P}\left[\tilde{A}+\tilde{B} \tilde{K}+\Delta_{\tilde{A}}+\Delta_{\tilde{B}} \tilde{K}\right], \\
\tilde{H}_{3}=\left[\tilde{A}_{d}+\Delta_{\tilde{A}_{d}}\right]^{T} \tilde{P}\left[\tilde{A}_{d}+\Delta_{\tilde{A}_{d}}\right]-\tilde{W}
\end{gathered}
$$

and $\tilde{P}>0$ is an associated cost matrix for the system $\tilde{\mathbf{S}}$. It is easy to prove using (7) and assuming $M V=0, N=0$, $M_{d} V=0, V^{T} M_{Q^{*}} V=0$ and $N_{R^{*}}=0$ that the inequality matrix (16) is equivalent to (11). Thus, $u(k)=\tilde{K} \tilde{x}(k)$ is a quadratic guaranteed cost controller for $\tilde{\mathbf{S}}$ if and only if $u(k)=K x(k)=\tilde{K} V x(k)$ is a quadratic guaranteed cost controller for $\mathrm{S}$. Obviously, $P=V^{T} \tilde{P} V$ is a symmetric definite-positive matrix if $\tilde{P}$ is also a symmetric definite-positive matrix. The same way of reasoning holds for the semidefinite-positive matrix $W$. The equality $J_{0}=\tilde{J}_{0}$ follows from (14) taking into account that $X^{-1}=P$. Thus,

$$
\begin{aligned}
J_{0} & =x^{T}(0) X^{-1} x(0)+\sum_{k=-d}^{-1} \phi^{T}(k) X^{-1} T X^{-1} \phi(k) \\
& =x^{T}(0) V^{T} \tilde{P} V x(0)+\sum_{k=-d}^{-1} \phi^{T}(k) V^{T} \tilde{P} V T V^{T} \tilde{P} V \phi(k) \\
& =\tilde{x}^{T}(0) \tilde{P} \tilde{x}(0)+\sum_{k=-d}^{-1} \tilde{\phi}^{T}(k) \tilde{P} \tilde{T} \tilde{P} \tilde{\phi}(k)=\tilde{J}_{0} .
\end{aligned}
$$

\subsection{Decentralized Control}

Let us specialize the above results on the decentralized control design. Suppose that the closed-loop system is designed under the presence of information structure constraints on feedback control. Consider the basic illustrative structures with two overlapping subsystems 
for the matrices $A, \Delta_{A}, A_{d}, \Delta_{A_{d}}$ and $B, \Delta_{B}$, respectively. They have the following forms:

$$
\left(\begin{array}{cc:c}
A_{11} & A_{12} & A_{13} \\
\hdashline A_{21} & A_{22} & A_{23} \\
\hdashline A_{31} & A_{32} & A_{33}
\end{array}\right), \quad\left(\begin{array}{c:c}
B_{11} & B_{12} \\
B_{21} & B_{22} \\
\hdashline B_{31} & B_{32}
\end{array}\right)
$$

This structure has been extensively adopted as a prototype structure (Ikeda and Šiljak 1986), (Ikeda $e$ al. 1981), (Šiljak 1991). Consider the above overlapping structure of subsystems in the original system. A standard particular choice of the transformation matrix $V$ is given by

$$
V=\left(\begin{array}{ccc}
I_{n_{1}} & 0 & 0 \\
0 & I_{n_{2}} & 0 \\
0 & I_{n_{2}} & 0 \\
0 & 0 & I_{n_{3}}
\end{array}\right)
$$

This particular selection of $V$ is chosen to lead in a simple natural way to an expanded system. The component $x_{2}$ of the vector $x^{T}=\left(x_{1}^{T}, x_{2}^{T}, x_{3}^{T}\right)$ appears doubled in $\tilde{x}^{T}$. The dimensions of the components $\left(x_{1}^{T}, x_{2}^{T}, x_{3}^{T}\right)$ are $n_{1}, n_{2}, n_{3}$ satisfying $n_{1}+n_{2}+n_{3}=n$, respectively. The partition of $u^{T}=\left(u_{1}^{T}, u_{2}^{T}\right)$ has two components of dimensions $m_{1}, m_{2}$ such that $m_{1}+m_{2}=m$. It means that the decentralized controller designed in the expanded state space is a block diagonal matrix with two subblocks of dimensions $m_{1} \times\left(n_{1}+n_{2}\right)$ and $m_{2} \times\left(n_{2}+n_{3}\right)$ of the gain matrix. It has the following form:

$$
\tilde{K}_{D}=\left(\begin{array}{cc:cc}
\tilde{K}_{11} & \tilde{K}_{12} & 0 & 0 \\
\hdashline 0 & 0 & \tilde{K}_{23} & \tilde{K}_{24}
\end{array}\right) .
$$

The corresponding contracted gain matrix is given by

$$
K_{D}=\left(\begin{array}{c:c:c}
\tilde{K}_{11} & \tilde{K}_{12} & 0 \\
\hdashline 0 & \tilde{K}_{23} & \tilde{R}_{24}
\end{array}\right) \text {. }
$$

Following this standard way of reasoning, it is suitable to introduce an another definition. Consider only the system (1), (2) with $K=K_{D}$ in (12).

Definition 10. Suppose the system (1) with cost function (2). A state feedback control law $u(k)=K_{D} x(k)$, with $K_{D}$ as given in (21), is said to be a quadratic guaranteed cost controller with associated cost matrix $P>0$ for the system (1), (5) if there exists a semipositivedefinite matrix $S$ such that (11)-(12) are satisfied.

Theorem 11. Consider the systems (1) and (3) with their corresponding cost functions (2) and (4), respectively. Consider the subsystem structure (18) and the transformation matrix (19). Suppose that $M V=0$, $N=0, M_{d} V=0, V^{T} M_{Q} V=0$ and $N_{R^{*}}=0$ hold. Then, $\tilde{u}(k)=\tilde{K}_{D} \tilde{x}(k)$ is a quadratic guaranteed cost controller with a cost matrix $\tilde{P}>0$ for the system $\tilde{S}$ if and only if $u(k)=K_{D} x(k)$ is a quadratic guaranteed cost controller with a cost matrix $P>0$ for $S$ and $J_{0}=\tilde{J}_{0}$.

Proof. It is straightforward because this theorem is a particular case of Theorem 9.
The results are presented only for the structure with two overlapping subsystems. However, they can be directly generalized for any number of interconnected overlapping subsystems.

\section{EXAMPLE}

\subsection{Problem Statement}

Consider the system (1) and the cost function (2) as follows:

$$
\begin{aligned}
A & =\left(\begin{array}{ccc}
1 & 0 & 0 \\
1 & 0 & -1 \\
0 & 1 & 1
\end{array}\right), \quad B=\left(\begin{array}{ll}
1 & 0 \\
0 & 1 \\
1 & 0
\end{array}\right), \\
A_{d} & =D=E_{1}=E_{d}=\left(\begin{array}{ccc}
0.1 & 0 & 0 \\
0 & 0.1 & 0 \\
0 & 0 & 0.1
\end{array}\right), \\
E_{2} & =\left(\begin{array}{cc}
0.1 & 0 \\
0 & 0 \\
0 & 0.1
\end{array}\right), Q^{*}=\operatorname{diag}\{1,2,1\}, \\
R^{*} & =\operatorname{diag}\{1,1\}, x(0)=\left(\begin{array}{l}
1 \\
0 \\
0
\end{array}\right), x(-1)=\left(\begin{array}{l}
0 \\
1 \\
0
\end{array}\right), \\
d & =1 .
\end{aligned}
$$

Find the overlapping quadratic guaranteed cost controller for the above system, where two overlapping subsystems are supposed with the dimensions $n_{1}=$ $n_{2}=n_{3}=1$ by (18). Compare these results with the case of the centralized control design serving as a reference. Use the delay independent LMI approach for the control design.

\subsection{Results}

Decentralized controller. Consider the expansion of the system $\mathbf{S}$ with the transformation $V$ given in (19), as follows:

$$
V=\left(\begin{array}{lll}
1 & 0 & 0 \\
0 & 1 & 0 \\
0 & 1 & 0 \\
0 & 0 & 1
\end{array}\right) .
$$

Suppose that the complementary matrix $M$ has the following structure:

$$
M=\left(\begin{array}{llll}
0 & m_{12} & -m_{12} & 0 \\
0 & m_{22} & -m_{22} & 0 \\
0 & m_{32} & -m_{32} & 0 \\
0 & m_{42} & -m_{42} & 0
\end{array}\right),
$$

where $m_{i 2}, i=1, \cdots, 4$, are free parameters . The structure of the matrix $M$ allow to construct different expanded systems but all with the same cost (Bakule et al. 2000a), (Bakule et al. 2000b). The remaining complementary matrices are selected as $M_{d}=0$, $N=0, M_{Q^{*}}=0, N_{R^{*}}=0$. Therefore, the weighting matrices $\tilde{Q}^{*}=I$ and $\tilde{R}^{*}=R^{*}=I$. Such choice ensures that the results presented in Section 3 are satisfied. Denote $\tilde{X}_{D}$, $\tilde{T}_{D}$ and $\tilde{Y}_{D}$ block diagonal matrices and consider them as the matrices $X, T$ and $Y$ appearing in Proposition 6 . To obtain the structure (20) for the gain matrix $\tilde{K}_{D}$, we must impose some conditions on the structure of the matrices $\tilde{X}_{D}$, and $\tilde{Y}_{D}$. They are as follows:

$$
\tilde{X}_{D}=\left(\begin{array}{cccc}
x_{11} & x_{12} & 0 & 0 \\
x_{12} & x_{22} & 0 & 0 \\
0 & 0 & x_{33} & x_{34} \\
0 & 0 & x_{34} & x_{44}
\end{array}\right), \tilde{Y}_{D}=\left(\begin{array}{cccc}
y_{11} & y_{12} & 0 & 0 \\
0 & 0 & y_{23} & y_{24}
\end{array}\right) \text {. }
$$


By applying the LMI design by (13) on this expanded system $\tilde{\mathbf{S}}$, we get the gain matrix

$$
\tilde{K}_{D}=\left(\begin{array}{cccc}
-0.3515 & -0.3782 & 0 & 0 \\
0 & 0 & -0.3972 & 0.2086
\end{array}\right) .
$$

The corresponding contracted gain matrix has the following form

$$
K_{D}=\left(\begin{array}{ccc}
-0.3515 & -0.3782 & 0 \\
0 & -0.3972 & 0.2086
\end{array}\right) .
$$

The associated bound on the cost equals to $J \leqslant J_{0}=20.94$. Centralized controller: The direct computation on the original system and cost results in the following controller:

$$
K=\left(\begin{array}{rrr}
-0.5315 & -0.3135 & -0.3105 \\
-0.2753 & -0.6925 & 0.2700
\end{array}\right)
$$

with the bound on the cost equal to $J \leqslant J_{0}=9.03$.

The centralized control design case serves only as a reference to compare the bounds on costs in both cases. The upper bound $J_{0}$ is greater than in the centralized case because of given structural constraints as expected. All computations have been performed using Matlab LMI Control Toolbox (Gahinet et al. 1995).

\section{CONCLUSION}

The paper presents the expansion-contraction relations within the framework of the quadratic guaranteed cost control problem when considering a class of nonlinear but nominally linear uncertain state delayed discrete-time systems. Unknown arbitrarily time-varying norm bounded uncertainties with known bounds are considered. It contributes by the conditions under which these relations hold for the closedloop systems including the requirement on the equality of bounds on the costs. The controller design is performed for the expanded system using the delay independent linear matrix inequality (LMI) method. The results are specialized on the overlapping feedback control design under decentralized information structure constraints. A numerical illustrative example is supplied.

\section{REFERENCES}

Bakule, L., J. Rodellar and J.M. Rossell (2000a). Generalized selection of complementary matrices in the inclusion principle. IEEE Transactions on Automatic Control AC-45(6), 1237-1243.

Bakule, L., J. Rodellar and J.M. Rossell (2000b). Structure of expansion-contraction matrices in the inclusion principle for dynamic systems. SIAM Journal on Matrix Analysis and Applications 21(4), 1136-1155.

Bakule, L., J. Rodellar and J.M. Rossell (2002a). Overlapping guaranteed cost control for timevarying discrete-time uncertain systems. In: Proceedings of the American Control Conference. Anchorage, Alaska, USA. pp. 1705-1710.
Bakule, L., J. Rodellar and J.M. Rossell (2002b). Overlapping quadratic optimal control of linear time-varying commutative systems. SIAM Journal on Control and Optimization 40(5), 16111627.

Bakule, L., J. Rodellar, J.M. Rossell and P. Rubió (2001). Preservation of controllability-observability in expanded systems. IEEE Transactions on Automatic Control AC-46(7), 1155-1162.

Gahinet, P., A. Nemirovski, A. Laub and M. Chilali (1995). The LMI Control Toolbox. The MathWorks, Inc.

Guan, X., Z. Lin and G. Duan (1999). Robust guaranteed cost control for discrete-time uncertain systems with delay. In: IEE Proceedings - Control Theory and Applications. number 6. pp. 598602 .

Ikeda, M. and D.D. Šiljak (1980). Overlapping decompositions, expansions and contractions of dynamic systems. Large Scale Systems 1(1), 29-38.

Ikeda, M. and D.D. Siljak (1986). Overlapping decentralized control with input, state, and output inclusion. Control-Theory and Advanced Technologv 2(2), 155-172.

Ikeda, M., D.D. Šiljak and D.E. White (1981). Decentralized control with overlapping information sets. Journal of Optimization Theory and Applications 34(2), 279-310.

Ikeda, M., D.D. Šiljak and D.E. White (1984). An inclusion principle for dynamic systems. IEEE Transactions on Automatic Control AC29(3), 244-249.

Jiang, P., H. Su and J. Chu (2000). LMI approach to optimal guaranteed cost control for a class of linear uncertain discrete systems. In: Proceedings of the American Control Conference. Chicago, Illinois, USA. pp. 327-331.

Šiljak, D.D. (1991). Decentralized Control of Complex Systems. Academic Press. New York, USA.

Šiljak, D.D. and D.M. Stipanović (2000). Robust stabilization of nonlinear systems: The LMI approach. Mathematical Problems in Engineering 6, 461-493.

Xie, L. and Y.Ch. Soh (1993). Control of uncertain discrete-time systems with guaranteed cost. In: Proceedings of the 32nd. Conference on Decision and Control. San Antonio, Texas, USA. pp. 5661.

Yu, L. (1997). Guaranteed cost control of uncertain linear discrete-time systems. In: Proceedings of the American Control Conference. Alburquerque, New Mexico, USA. pp. 3181-3184.

Yu, L., F. Gao and A. Xue (2000). Guaranteed cost control of uncertain discrete linear timedelay systems. In: Proceedings of the American Control Conference. Chicago, Illinois, USA. pp. 2481-2485. 